\title{
Integrated Chinese Herbal Medicine and Western Medicine on the Survival in Patients with Colorectal Cancer: A Retrospective Study of Medical Records
}

\author{
Ming-Hsien Yeh, ${ }^{1,2}$ Hung-Pin Chiu, ${ }^{3}$ Mei-Chun Wu, ${ }^{3}$ Malcolm Koo (iD, ${ }^{4,5}$ Nai-Wei Lin (D), \\ Kou-Kai Liao, ${ }^{3}$ Chia-Chou Yeh $\mathbb{D}^{2,7}$ and Te-Mao Li $\mathbb{B D}^{1}$ \\ ${ }^{1}$ Graduate Institute of Chinese Medicine, China Medical University, Taichung 40402, Taiwan \\ ${ }^{2}$ Department of Chinese Medicine, Dalin Tzu Chi Hospital, Buddhist Tzu Chi Medical Foundation, Dalin, Chiayi 62247, Taiwan \\ ${ }^{3}$ Department of Information Management, Nanhua University, Dalin, Chiayi 62249, Taiwan \\ ${ }^{4}$ Graduate Institute of Long-term Care, Tzu Chi University of Science and Technology, Hualien 97005, Taiwan \\ ${ }^{5}$ Dalla Lana School of Public Health, University of Toronto, Toronto, Ontario M5T 3M7, Canada \\ ${ }^{6}$ Institute of Computer Science and Information Engineering, National Chung Cheng University, Minxiong, Chiayi 621, Taiwan \\ ${ }^{7}$ School of Post-Baccalaureate Chinese Medicine, Tzu Chi University, Hualien 97004, Taiwan
}

Correspondence should be addressed to Chia-Chou Yeh; yehcc0530@gmail.com and Te-Mao Li; leedemaw@mail.cmu.edu.tw

Received 20 July 2019; Accepted 6 December 2019; Published 13 January 2020

Academic Editor: Jian-Li Gao

Copyright (c) 2020 Ming-Hsien Yeh et al. This is an open access article distributed under the Creative Commons Attribution License, which permits unrestricted use, distribution, and reproduction in any medium, provided the original work is properly cited.

\begin{abstract}
Recent studies suggested that Traditional Chinese Medicine could play a beneficial role in conventional cancer treatment. The aim of this retrospective cohort study was to investigate the effect of Chinese herbal medicine (CHM) combined with Western medicine on the survival of patients with colorectal cancer. A retrospective cohort study was conducted on patients with newly diagnosed colorectal cancer identified from the Dalin Tzu Chi Hospital Cancer Registry Database in 2004-2014. Combining with the medical records of the study hospital, patients were classified into CHM users and CHM nonusers. Kaplan-Meier analyses and Cox proportional hazards regression analyses were used to investigate the survival between CHM users and CHM nonusers. A total of 535 patients with colorectal cancer were included in the study with 147 of them were CHM users. The log-rank test for Kaplan-Meier survival curve revealed a statistically significant difference between the survival of CHM users and CHM nonusers $(\mathrm{P}=0.006)$. Results from multivariate Cox regression analysis showed that CHM use was significantly associated with better survival (adjusted hazard ratio $=0.54,95 \% \mathrm{CI}=0.38$ to 0.77 ). In addition, the CHM formulae Jia Wei Xiao Yao San, Zhi Bah Di Huang Wan, Ping Wei San, and Qui Pi Tang were significantly associated with better survival. In conclusion, findings from this retrospective cohort study indicated that integrated CHM and Western medicine could improve survival in patients with colorectal cancer. Additional research on integrating TCM with Western medicine to improve cancer survival is warranted.
\end{abstract}

\section{Introduction}

Colorectal cancer is a leading cause of cancer death worldwide and is the third leading cause of cancer mortality in Taiwan [1]. While the overall five-year survival rate of colorectal cancer has exceeded $65 \%$ in most affluent countries [2], it is only $9-12 \%$ for patients with stage IV colorectal cancer $[3,4]$. Currently, the mainstream treatment of colorectal cancer is laparoscopic surgical resection for primary disease, but radiotherapy and chemotherapy are often required for metastatic disease [5]. In addition to Western medical treatments, Traditional Chinese Medicine (TCM) has been used in the treatment of patients with cancer [6]. TCM has been reported to be able to alleviate chemotherapy nausea [7], reduce pain [8], improve the curative effect of cancer treatment, enhance quality of life, and reduce adverse events [9]. Research indicated that certain Chinese herbal medicines (CHM), such as Salvia 
miltiorrhiza, could inhibit the proliferation of colorectal cancer cells by apoptosis [10], thereby effectively prolonging survival [11-14].

A secondary analysis of the Taiwan's National Health Information Research Database (NHIRD), a nationwide, population-based medical claim records database, showed that Xiang Sha Liu Jun Zi Tang (7.1\%), Bu Zhong Yi Qi Tang (4.3\%), and Jia Wei Xiao Yao San (4.1\%) were the top three most commonly prescribed single Chinese herbal medicine (CHM) formulae prescribed for postsurgery colon cancer patients [11]. However, the study did not analyze the effect of these formulae on survival rates. In addition, the NHIRD did not contain information on cancer stage, chemotherapy, radiotherapy, and surgery [15], and hence their potential confounding effects could not be controlled. Alternatively, the use of the Taiwan Cancer Registry (TCR) could overcome this data limitation [16]. The aim of this retrospective cohort study was to investigate the effect of CHM combined with Western medicine compared with Western medicine alone on the survival of patients with colorectal cancer, using local hospital medical record data linked with the TCR. In addition, the effectiveness of the 10 most commonly used $\mathrm{CHM}$ formulae with different dosages on survival was investigated.

\section{Materials and Methods}

2.1. Data Source. The data sources of this study comprised the TCR of Dalin Tzu Chi Hospital [16] and medical records database of the study hospital from 2004 to 2014. First, we used TCR to identify patients with newly diagnosed colorectal cancer. Their treatment, clinical stage, and cause of death were also obtained from the TCR. Only deaths due to colorectal cancer were considered as the mortality outcome in this study. Diagnosis codes for cancer types were based on the ICD for Oncology, 3rd edition (ICD-O-3), from 2002. The medical records of the study hospital were used to ascertain basic and clinical characteristics of the patients and also the type, dosage, frequency, and treatment duration of $\mathrm{CHM}$ used. Disease diagnoses were based on the International Classification of Diseases, Ninth Revision, Clinical Modification (ICD-9-CM). Study participants had to be treated for at least 30 days at Dalin Tzu Chi Hospital to be eligible for the study. The study protocol was approved by the institutional review board of Dalin Tzu Chi Hospital, Buddhist Tzu Chi Medical Foundation (IRB no. B10503013).

2.2. Study Design and Exposure Assessment. We conducted a retrospective cohort study to examine the association of $\mathrm{CHM}$ use and survival rates in patients with colorectal cancer. To avoid immortal time bias, patients were followed starting from 3 months after their first colorectal cancer diagnosis [17] to the date of death or the end of the study period, whichever came first. A total of 1209 patients with colorectal cancer (ICD-9-CM: 153-154.8 and ICD-O-3: C18.0-C21.8) between 2004 and 2014 were identified from the TCR. We excluded patients whose (1) survival status was unclear or death reason was unknown (code number: 7777 or 7798), or (2) cancer stage unknown or stage 0. A total of 535 eligible patients were included.

Study patients were classified as either CHM users or CHM nonusers based on whether their cumulative days of $\mathrm{CHM}$ use were more than 30 days during the first year following the first diagnosis date of their colorectal cancer. $\mathrm{CHM}$ use was considered as interrupted when the interval between two consecutive CHM treatments exceeded six weeks.

2.3. Outcome Assessment. The primary outcome of this study was colorectal cancer-specific survival. Survival time was calculated in months from the first date of colorectal cancer diagnosis to either death or the end of the study period. Potential confounding variables included age, sex, comorbidities at baseline, clinical stage of cancer, and treatment of colorectal cancer (surgery, radiotherapy, and chemotherapy). According to the National Health Insurance (NHI), the mortality rate of colorectal cancer increases considerably after the age of 45 years. Therefore, the patients were stratified into three subgroups by age ( $\leq 45$ years, $46-65$ years, and $>65$ years) in all the analyses.

Based on individual medical records one year before initial cohort entry, the Charlson-Deyo comorbidity index was used to assess the presence of comorbidity [18]. Following the classification system developed by the American Joint Committee on Cancer (AJCC 7th edition), clinical stage was assigned I, II, III, or IV. Uncertain information (code number: 888 or 999 ) was regarded as an unknown stage. Metastasis was recorded as yes or no.

For a specific CHM, the total dosage taken by each patient was calculated by multiplying days, doses, and frequencies of treatments. Based on the overall dose of medication, we obtained the top 10 commonly used CHM. For each of the $10 \mathrm{CHM}$ formulae, patients taking dosage higher than the median were classified as the high-dose group, otherwise as the low-dose group.

2.4. Statistical Analysis. We used Chi-squared tests to compare the proportions of demographic variables, clinical stage of cancer, comorbidity variables, and treatment methods (surgery, chemotherapy, and radiotherapy) in the two groups. The Kaplan-Meier survival curve and log-rank test were used to compare survival curves. Univariate and multivariate Cox proportional hazards regression analyses were used to examine the hazard ratio (HR) of survival for CHM use and for dosage used. The multivariate-adjusted model was developed using backward elimination based on the likelihood ratio test. All $P$ values less than 0.05 were considered statistically significant.

\section{Results}

3.1. Characteristics of Patients with Colorectal Cancer. The study identified 535 colorectal cancer patients; 388 were CHM nonusers, and 147 were CHM users. Table 1 shows a comparison of age and sex distribution of the source population (1,209 patients) with our final study sample (535 
TABLE 1: Comparison of age and sex distribution of the 1,209 patients with colorectal cancer identified from the Taiwan Cancer Registry and the 535 patients included in this study.

\begin{tabular}{|c|c|c|c|c|c|}
\hline \multirow[t]{2}{*}{ Variable } & \multicolumn{2}{|c|}{$\begin{array}{l}\text { Source population } \\
\qquad(N=1209)\end{array}$} & \multicolumn{2}{|c|}{ Study sample $(N=535)$} & \multirow[t]{2}{*}{$P$} \\
\hline & No. & $\%$ & No. & $\%$ & \\
\hline Age (years) & & & & & 0.110 \\
\hline$\leq 45$ & 93 & 7.7 & 43 & 8.0 & \\
\hline $46-65$ & 442 & 36.5 & 192 & 35.9 & \\
\hline$\geq 66$ & 674 & 55.7 & 300 & 56.1 & \\
\hline Sex & & & & & 0.969 \\
\hline Male & 643 & 53.2 & 284 & 53.1 & \\
\hline Female & 566 & 46.8 & 251 & 46.9 & \\
\hline
\end{tabular}

patients). The distribution of sex and age group was not significantly different between the source population and our final sample.

Table 2 indicates that clinical stage, age, sex, treatment (surgery, radiotherapy, and chemotherapy), distant metastases, comorbidity score 2, and comorbidity score 6 did not differ significantly between CHM users and nonusers. However, the proportion of comorbidity score 1 in $\mathrm{CHM}$ users is considerably higher than that in CHM nonusers $(P=0.010)$.

3.2. Survival by CHM Use. The mean follow-up period for all 535 patients was 36.0 months $(\mathrm{SD}=22.8)$. The mean followup period for the CHM nonusers was 35.2 months $(\mathrm{SD}=23.9)$, ranging between 3.0 and 90.9 months; the mean follow-up period for the CHM users was 38.1 months $(\mathrm{SD}=19.7)$, ranging between 3.5 and 91.4 months. Overall, 197 deaths (36.8\%) occurred during the study period. The CHM nonusers group had 156 deaths (40.2\%), and the CHM users group had 41 deaths $(27.9 \%)$.

The log-rank test for Kaplan-Meier survival curves revealed a statistically significant difference between the survival of the two groups $(P=0.006)$ (Figure 1$)$. The survival rates of the first, third, and fifth years for $\mathrm{CHM}$ nonusers were $80 \%, 62 \%$, and $54 \%$, while for $\mathrm{CHM}$ users were $92 \%, 75 \%$, and $63 \%$.

Further analyses revealed that there were no significant differences in the survival curves of stages I $(P=0.966)$ and II $(P=0.581)$ cancer (Figures $2(\mathrm{a})$ and $2(\mathrm{~b}))$, but there were significant differences in the survival curves of stages III $(P=0.027)$ and IV $(P=0.003)$ cancer (Figures $2(\mathrm{c})$ and $2(\mathrm{~d}))$.

3.3. Cox Regression Analysis of Patients with Colorectal Cancer. The univariate Cox regression analysis showed a significant association between the use of CHM and increased survival $(\mathrm{HR}=0.62,95 \% \mathrm{CI}=0.44-0.87, P=0.007)$ (Table 3). After adjusting for potential confounders, the use of CHM remained significantly associated with better survival (adjusted $\mathrm{HR}=0.54,95 \% \mathrm{CI}=0.38$ to $0.77, P=0.001$ ). Results of the multivariate analysis also revealed that the cancer stage was significantly associated with survival (stage 2-adjusted $\mathrm{HR}=2.98,95 \% \mathrm{CI}=1.44$ to $6.18, P=0.003$; stage 3 -adjusted $\mathrm{HR}=4.28,95 \% \mathrm{CI}=2.18$ to $8.42, P<0.001$; stage 4 -adjusted $\mathrm{HR}=17.49 ; 95 \% \mathrm{CI}=8.90$ to $34.38 ; P<0.001)$. In
TABle 2: Characteristics of patients with colorectal cancer $(N=535)$.

\begin{tabular}{|c|c|c|c|c|c|}
\hline \multirow[t]{2}{*}{ Variable } & \multicolumn{2}{|c|}{$\begin{array}{l}\text { CHM users } \\
(N=147)\end{array}$} & \multicolumn{2}{|c|}{$\begin{array}{c}\mathrm{CHM} \\
\text { nonusers } \\
(N=388)\end{array}$} & \multirow[t]{2}{*}{$P$} \\
\hline & No. & $\%$ & No. & $\%$ & \\
\hline Age (years) & & & & & 0.075 \\
\hline$\leq 45$ & 10 & 6.8 & 33 & 8.5 & \\
\hline $46-65$ & 64 & 43.5 & 128 & 33.0 & \\
\hline$\geq 66$ & 73 & 49.7 & 227 & 58.5 & \\
\hline Sex & & & & & 0.851 \\
\hline Male & 79 & 53.7 & 205 & 52.8 & \\
\hline Female & 68 & 46.3 & 183 & 47.2 & \\
\hline Clinical stage & & & & & 0.774 \\
\hline I & 30 & 20.4 & 71 & 18.3 & \\
\hline II & 22 & 15.0 & 67 & 17.3 & \\
\hline III & 64 & 43.5 & 158 & 40.7 & \\
\hline IV & 31 & 21.1 & 92 & 23.7 & \\
\hline \multicolumn{6}{|l|}{ Treatment } \\
\hline Surgery & 137 & 93.2 & 359 & 92.5 & 0.790 \\
\hline Radiotherapy & 49 & 33.3 & 105 & 27.1 & 0.153 \\
\hline Chemotherapy & 115 & 78.2 & 273 & 70.4 & 0.069 \\
\hline Distant metastases & 31 & 21.1 & 92 & 23.7 & 0.520 \\
\hline Comorbidity score 1 & 18 & 12.2 & 22 & 5.7 & 0.010 \\
\hline Comorbidity score 2 & 8 & 5.4 & 20 & 5.2 & 0.894 \\
\hline Comorbidity score 6 & 9 & 6.1 & 18 & 4.6 & 0.659 \\
\hline
\end{tabular}

CHM:Chinese herbal medicine. Comorbidity score 1: myocardial infarction, congestive heart failure, peripheral vascular disease, cerebrovascular disease, dementia, chronic pulmonary disease, rheumatologic disease, peptic ulcer disease, mild liver disease, and diabetes. Comorbidity score 2: diabetes with chronic complication, hemiplegia or paraplegia, renal disease, and malignancy (including leukemia and lymphoma). Comorbidity score 6: metastases solid tumor and acquired immune deficiency syndrome.

addition, surgery and chemotherapy were associated with better survival with adjusted HRs of 0.29 (95\% CI $=0.19$ to $0.43 ; P<0.001)$ and $0.47(95 \% \mathrm{CI}=0.34$ to $0.65 ; P<0.001)$, respectively.

3.4. Top 10 Commonly Used CHM on Survival. Based on the total dosage of $147 \mathrm{CHM}$ users, the top 10 used herb formulae are presented in Table 4. As shown in Table 5, certain Chinese herbal formulae were found to be associated with better survival. Univariate Cox hazards analyses showed that the use of high doses of Jia Wei Xiao Yao San $(\mathrm{HR}=0.34,95 \% \mathrm{CI}=0.14$ to $0.82, P=0.017)$ and Liu Wei 


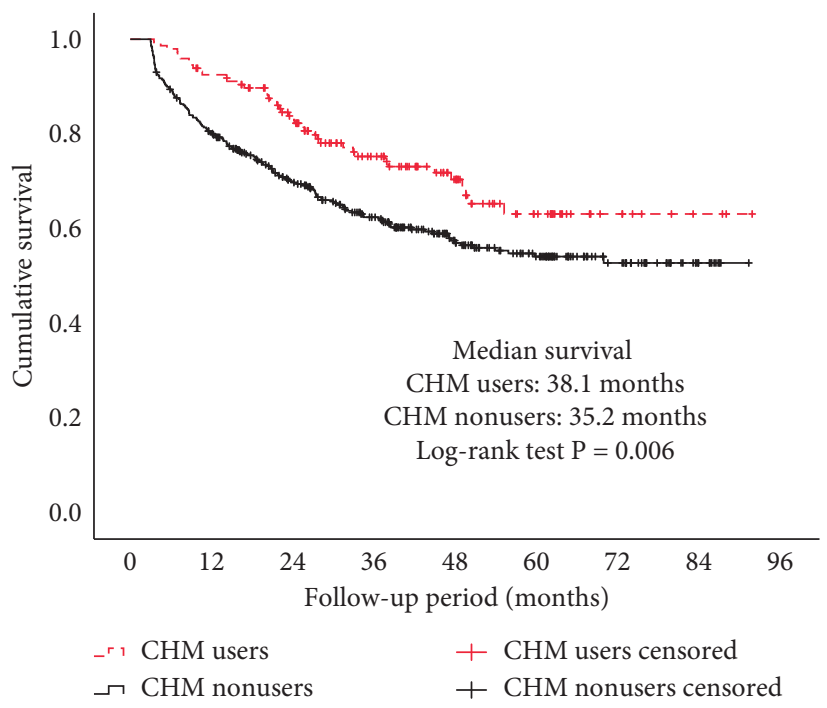

Figure 1: Kaplan-Meier survival curves for patients with colorectal cancer by Chinese herbal medicine (CHM) use.

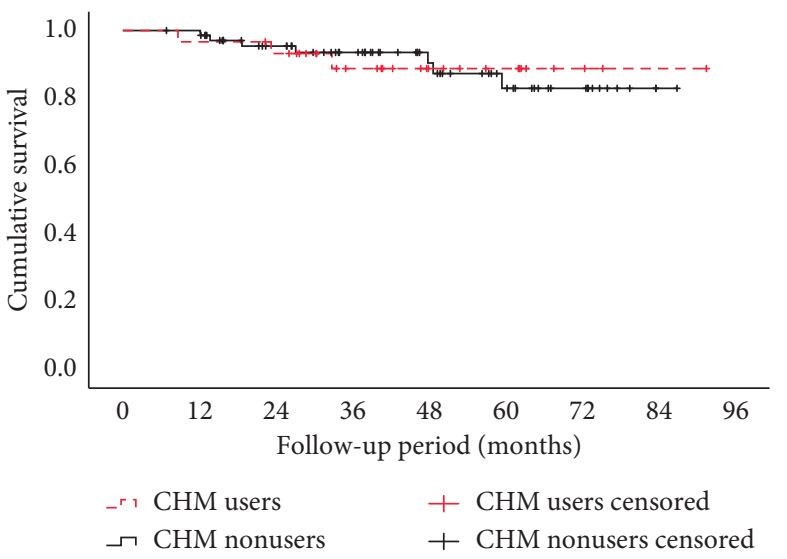

(a)

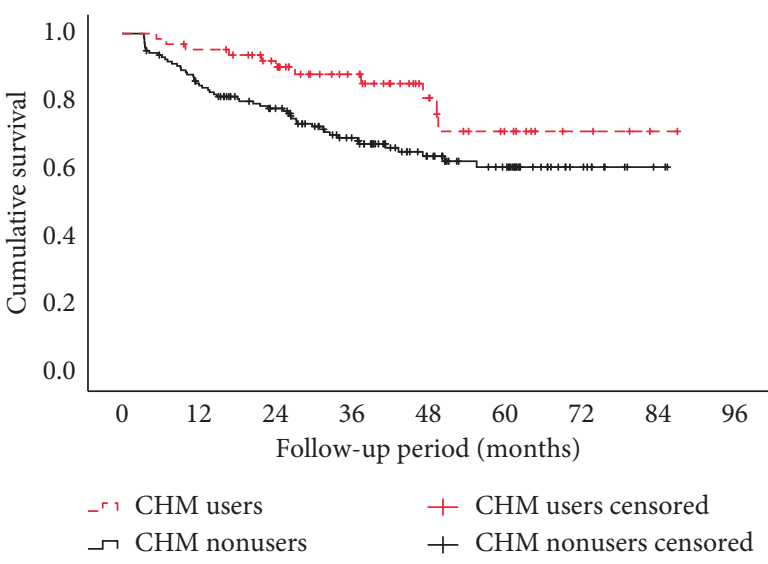

(c)

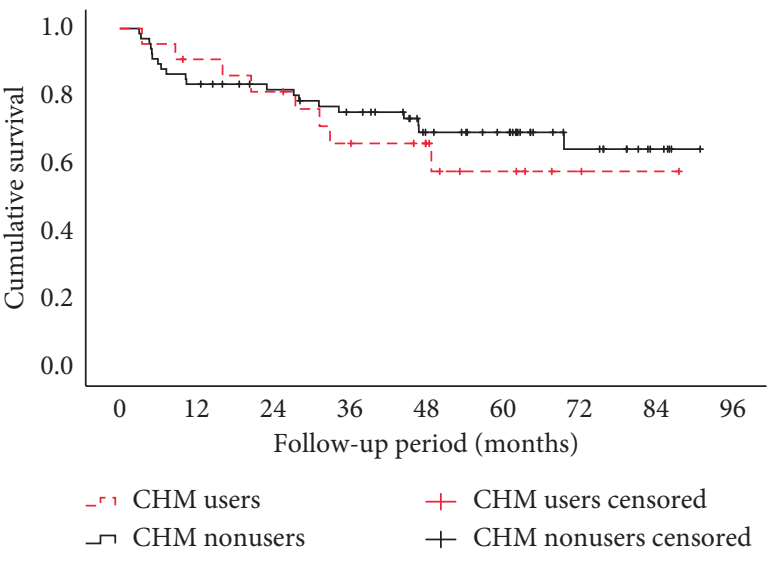

(b)

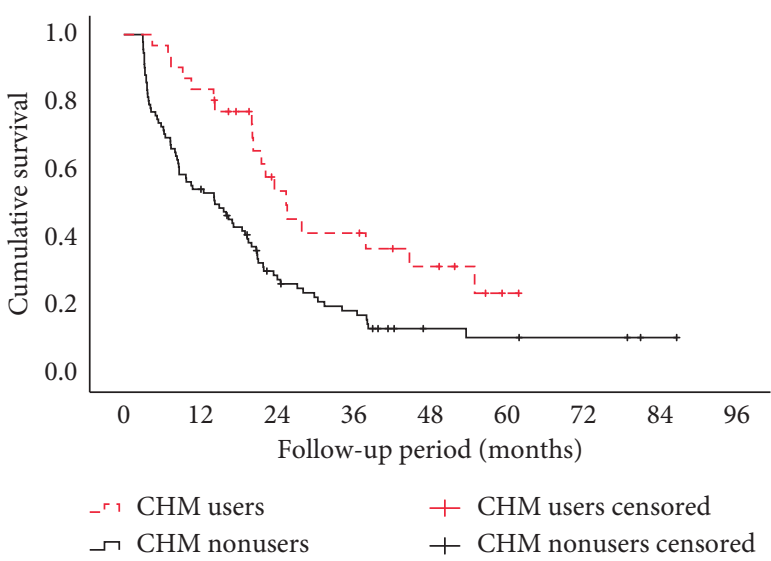

(d)

FIGURE 2: Kaplan-Meier survival curves for patients with colorectal cancer by Chinese herbal medicine use: (a) stage (I), (b) stage II, (c) stage III, and (d) stage IV.

Di Huang Wan $(\mathrm{HR}=0.12,95 \% \mathrm{CI}=0.02$ to 0.89 , $P=0.038$ ) was significantly associated with better survival. After adjusting for potential confounding factors, the use of high doses of Jia Wei Xiao Yao San (adjusted HR=0.38, $95 \% \mathrm{CI}=0.16$ to $0.93, P=0.035)$, Ban Xia Xie Xin Tang (adjusted $\mathrm{HR}=0.32,95 \% \mathrm{CI}=0.10$ to $1.00, P=0.049$ ), $\mathrm{Ping}$ 
TABLE 3: Survival of patients with colorectal cancer $(N=535)$.

\begin{tabular}{|c|c|c|c|c|c|c|}
\hline \multirow{2}{*}{ Variable } & \multicolumn{3}{|c|}{ Unadjusted analysis } & \multicolumn{3}{|c|}{ Adjusted analysis } \\
\hline & HR & $95 \% \mathrm{CI}$ & $P$ & Adjusted HR & $95 \% \mathrm{CI}$ & $P$ \\
\hline \multicolumn{7}{|c|}{ Chinese herbal medicine use } \\
\hline Nonusers $(<30 \mathrm{~d})$ & 1.00 & & & 1.00 & & \\
\hline Users $(\geq 30 \mathrm{~d})$ & 0.62 & $0.44-0.88$ & 0.007 & 0.54 & $0.38-0.77$ & 0.001 \\
\hline \multicolumn{7}{|l|}{ Age (years) } \\
\hline$\leq 45$ & 1.00 & & & - & - & - \\
\hline $46-65$ & 0.52 & $0.31-0.88$ & 0.014 & - & - & - \\
\hline$>65$ & 0.95 & $0.59-1.52$ & 0.820 & - & - & - \\
\hline \multicolumn{7}{|l|}{ Sex } \\
\hline Female & 1.00 & & & - & - & - \\
\hline Male & 1.29 & $0.97-1.72$ & 0.076 & - & - & - \\
\hline \multicolumn{7}{|l|}{ Clinical stage } \\
\hline I & 1.00 & & & 1.00 & & \\
\hline II & 3.25 & $1.58-6.70$ & 0.001 & 2.98 & $1.44-6.18$ & 0.003 \\
\hline III & 3.30 & $1.69-6.42$ & $<0.001$ & 4.28 & $2.18-8.42$ & $<0.001$ \\
\hline IV & 15.26 & $7.93-29.36$ & $<0.001$ & 17.49 & $8.90-34.38$ & $<0.001$ \\
\hline Surgery & 0.18 & $0.12-0.26$ & $<0.001$ & 0.29 & $0.19-0.43$ & $<0.001$ \\
\hline Radiotherapy & 0.82 & $0.60-1.12$ & 0.215 & - & - & - \\
\hline Chemotherapy & 0.76 & $0.56-1.02$ & 0.069 & 0.47 & $0.34-0.65$ & $<0.001$ \\
\hline Distant metastases & 5.69 & $4.27-7.57$ & $<0.001$ & - & - & - \\
\hline Comorbidity score 1 & 1.04 & $0.61-1.75$ & 0.896 & - & - & - \\
\hline Comorbidity score 2 & 0.59 & $0.28-1.24$ & 0.165 & - & - & - \\
\hline Comorbidity score 6 & 1.48 & $0.86-2.55$ & 0.161 & - & - & - \\
\hline
\end{tabular}

CI: confidence interval; HR: hazards ratio. Comorbidity score 1: myocardial infarction, congestive heart failure, peripheral vascular disease, cerebrovascular disease, dementia, chronic pulmonary disease, rheumatologic disease, peptic ulcer disease, mild liver disease, and diabetes. Comorbidity score 2: diabetes with chronic complication, hemiplegia or paraplegia, renal disease, and malignancy (including leukemia and lymphoma). Comorbidity score 6: metastases solid tumor and acquired immune deficiency syndrome.

TABLE 4: Top 10 commonly used Chinese herbal medicine formulae in patients with colorectal cancer $(N=147)$.

\begin{tabular}{|c|c|c|c|c|c|}
\hline Formula & Ingredients & Function & $\begin{array}{c}\text { No. of } \\
\text { patients }\end{array}$ & $\%$ & $\begin{array}{c}\text { Total dosage } \\
(\mathrm{g})\end{array}$ \\
\hline $\begin{array}{l}\text { Liu Wei Di Huang } \\
\text { Wan }\end{array}$ & $\begin{array}{c}\text { Radix Rehmanniae, Fructus corni } \\
\text { officinalis, Radix Dioscoreae Oppositae, } \\
\text { Paeonia suffruticosa Andr, Sclerotium } \\
\text { Poriae Cocos, Alismatis Rhizoma }\end{array}$ & Kidney yin deficient & 40 & 27.2 & 14,328 \\
\hline $\begin{array}{l}\text { Jia Wei Xiao Yao } \\
\text { San }\end{array}$ & $\begin{array}{l}\text { Rhizoma Atractylodis Macrocephalae, } \\
\text { Radix Glycyrrhizae Uralensis, Herba } \\
\text { Menthae Haplocalycis, Radix Angelicae } \\
\text { sinensis, Radix Paeoniae Lactiflorae, } \\
\text { Rhizoma zingiberis officinalis, Radix } \\
\text { Bupleuri, Cortex Moutan Radicis, } \\
\text { Fructus Gardeniae Jasminoides }\end{array}$ & Clears liver Qi stagnation and heat & 74 & 50.3 & 41,437 \\
\hline $\begin{array}{l}\text { Ban Xia Xie Xin } \\
\text { Tang }\end{array}$ & $\begin{array}{l}\text { Rhizoma Pinelliae Ternatae, Radix } \\
\text { Scutellariae Baicalensis, Radix Ginseng, } \\
\text { Radix Glycyrrhizae Uralensis, Rhizoma } \\
\text { zingiberis officinalis, Rhizoma Coptidis } \\
\text { (Huang Lian), Fructus Zizyphi Jujubae }\end{array}$ & $\begin{array}{l}\text { Harmonize the stomach and descend the } \\
\text { rebellious Qi }\end{array}$ & 45 & 30.6 & 17,617 \\
\hline Ping Wei San & $\begin{array}{c}\text { Atractylodes Rhizome, Magnolia Bark, } \\
\text { Dried Tangerine Peel, Licorice Root, } \\
\text { Fructus Zizyphi Jujubae }\end{array}$ & $\begin{array}{l}\text { Dries dampness and harmonizes spleen- } \\
\text { stomach relationship }\end{array}$ & 70 & 47.6 & 22,445 \\
\hline Zhi Gan Cao Tang & $\begin{array}{c}\text { Radix Glycyrrhizae Uralensis, Rhizoma } \\
\text { zingiberis officinalis, Ramulus } \\
\text { Cinnamomi Cassiae, Radix Ginseng, } \\
\text { Colla Corrii Asini, Tuber Ophiopogonis } \\
\text { Japonici, semen cannabis sativae, Fructus } \\
\text { Zizyphi Jujubae }\end{array}$ & Anemic and yang deficient syndrome & 31 & 21.1 & 12,498 \\
\hline
\end{tabular}


TABLE 4: Continued.

\begin{tabular}{|c|c|c|c|c|c|}
\hline Formula & Ingredients & Function & $\begin{array}{c}\text { No. of } \\
\text { patients }\end{array}$ & $\%$ & $\begin{array}{c}\text { Total dosage } \\
(\mathrm{g})\end{array}$ \\
\hline $\begin{array}{l}\text { Zhi Bai Di Huang } \\
\text { Wan }\end{array}$ & $\begin{array}{c}\text { Radix Rehmanniae, Fructus corni } \\
\text { officinalis, Radix Dioscoreae Oppositae, } \\
\text { Paeonia suffruticosa Andr., Sclerotium } \\
\text { Poriae Cocos, Alismatis Rhizoma, } \\
\text { Anemarrhena Rhizome, Cortex } \\
\text { Phellodendri }\end{array}$ & Yin deficient and heat is high & 34 & 23.1 & 13,274 \\
\hline Li Zhong Tang & $\begin{array}{l}\text { Rhizoma Atractylodis Macrocephalae, } \\
\text { Radix Ginseng, Radix Glycyrrhizae } \\
\text { Uralensis, Rhizoma zingiberis officinalis }\end{array}$ & Spleen deficient & 56 & 38.1 & 22,413 \\
\hline $\begin{array}{l}\text { Du Huo Ji Sheng } \\
\text { Tang }\end{array}$ & $\begin{array}{c}\text { Radix Angelicae Pubescentis, Ramulus } \\
\text { Sangjisheng, Cortex Eucommiae } \\
\text { Ulmoidis, Herba cum Radice Asari, Radix } \\
\text { Gentianae macrophylliae, Sclerotium } \\
\text { Poriae Cocos, Ramulus Cinnamomi } \\
\text { Cassiae, Radix Saposhnikoviae } \\
\text { divaricatae, Radix Ligustici Chuanxiong, } \\
\text { Radix Ginseng, Radix Glycyrrhizae } \\
\text { Uralensis, Radix Angelicae sinensis, } \\
\text { Radix Paeoniae Lactiflorae, Radix } \\
\text { Rehmanniae }\end{array}$ & $\begin{array}{c}\text { Gets rid of wind dampness, pain due to } \\
\text { Qi and blood stasis, tonic for Qi and } \\
\text { blood }\end{array}$ & 15 & 10.2 & 13,182 \\
\hline Qui Pi Tang & $\begin{array}{c}\text { Radix Ginseng, Sclerotium Poriae Cocos, } \\
\text { Rhizoma Atractylodis Macrocephalae, } \\
\text { Radix astragali, Semen Zizyphi Spinosae, } \\
\text { Arillus Euphoriae Longanae, Radix } \\
\text { Plygalae Tenuifoliae, Radix Glycyrrhizae } \\
\text { Uralensis, Fructus Zizyphi Jujubae, Radix } \\
\text { Aucklandiae, Rhizoma zingiberis } \\
\text { officinalis }\end{array}$ & Spleen deficient, blood deficient & 46 & 31.3 & 14,676 \\
\hline $\begin{array}{l}\text { Huo Xiang Zhen } \\
\text { Qi San }\end{array}$ & $\begin{array}{c}\text { Pericarpium Arecae Catechu, Radix } \\
\text { Angelicae Dahuricae, Folium Perillae } \\
\text { Frutescentis, Rhizoma Pinelliae Ternatae, } \\
\text { Pericarpium Citri Reticulatae, Cortex } \\
\text { Magnoliae Officinalis, Radix Platycodi } \\
\text { grandiflora, Herba Agastaches seu } \\
\text { Pogostemi, Radix Glycyrrhizae Uralensis }\end{array}$ & Relieves dampness & 54 & 36.7 & 14,821 \\
\hline
\end{tabular}

Wei San (adjusted $\mathrm{HR}=0.31,95 \% \mathrm{CI}=0.11$ to 0.84 , $P=0.022$ ), and Qui Pi Tang (adjusted $H R=0.26,95 \%$ $\mathrm{CI}=0.11$ to $0.59, P=0.001)$ was significantly associated with better survival. In addition, the use of low doses of Jia Wei Xiao Yao San was significantly associated with better survival (adjusted $\mathrm{HR}=0.40,95 \% \mathrm{CI}=0.20$ to 0.79 , $P=0.009$ ).

Table 6 shows the daily dosage and duration of administration for the four aforementioned significant CHM. The daily dosage ranged from $2.92 \mathrm{~g}$ for Ping Wei San to $4.68 \mathrm{~g}$ for Qui Pi Tang. The duration ranged from 25 days to 63 days for low and high doses of Jia Wei Xiao Yao San, respectively.

\section{Discussion}

In this retrospective cohort study, significant survival benefits for patients with colorectal cancer receiving treatment of integrated Chinese and Western medicine compared with Western medicine alone was observed, particularly among patients with stage III and IV cancer. Results from multivariate analysis, adjusting for cancer stage, surgery, and chemotherapy, showed that the hazards significantly decreased by $46 \%$ in CHM users compared with nonusers. Previous research also showed that the use of CHM could prolong the survival time of patients with colorectal cancer $[11,19]$.

Among the top 10 commonly used CHM, high doses of Jia Wei Xiao Yao San, Zhi Bah Di Huang Wan, Ping Wei San, and Qui Pi Tang were associated with better survival. To induce the therapeutic effect, daily dosages ranged between 2.92 and $4.68 \mathrm{~g}$, and treatment duration of 25 to 63 days was required. First, Jia Wei Xiao Yao San is generally the primary herb for treating cancers. It features emotional regulation, evacuation of Qi depression, and relief of gastrointestinal distress. The therapeutic effect of this herb could be through the alleviation of psychosomatic stress as a result of colon cancer treatment [20]. With reasonable doses, Jia Wei Xiao Yao San is safe and does not interact with 5-FU in conventional treatment [21].

Second, we found that Zhi Bah Di Huang Wan could significantly improve the survival patients with colorectal cancer. Patients with colon cancer and type II diabetes 
TABLE 5: Effects of 10 most commonly used Chinese herbal medicine formulae on the survival of patients with colorectal cancer $(N=535)$.

\begin{tabular}{|c|c|c|c|c|c|c|}
\hline \multirow{2}{*}{ Formula (n) } & \multicolumn{3}{|c|}{ Unadjusted analysis } & \multicolumn{3}{|c|}{ Adjusted analysis } \\
\hline & $\mathrm{HR}$ & $95 \% \mathrm{CI}$ & $P$ & Adjusted HR & $95 \% \mathrm{CI}$ & $P$ \\
\hline \multicolumn{7}{|c|}{ Jia Wei Xiao Yao San } \\
\hline Not used(476) & 1.00 & & & 1.00 & & \\
\hline Low dose (29) & 0.77 & $0.40-1.51$ & 0.446 & 0.40 & $0.20-0.79$ & 0.009 \\
\hline High dose (30) & 0.34 & $0.14-0.82$ & 0.017 & 0.38 & $0.16-0.93$ & 0.035 \\
\hline \multicolumn{7}{|c|}{ Liu wei Di huang Wan } \\
\hline Not used (503) & 1.00 & & & 1.00 & & \\
\hline Low dose (16) & 0.44 & $0.14-1.37$ & 0.158 & 0.64 & $0.20-2.01$ & 0.443 \\
\hline High dose (16) & 0.12 & $0.02-0.89$ & 0.038 & 0.18 & $0.02-1.28$ & 0.086 \\
\hline \multicolumn{7}{|c|}{ Zhi Bai Di Huang Wan } \\
\hline Not used (507) & 1.00 & & & 1.00 & & \\
\hline Low dose (15) & 0.74 & $0.30-1.79$ & 0.497 & 0.46 & $0.20-1.13$ & 0.091 \\
\hline High dose (13) & 0.15 & $0.02-1.09$ & 0.061 & 0.15 & $0.02-1.05$ & 0.056 \\
\hline \multicolumn{7}{|c|}{ Ban Xia Xie Xin Tang } \\
\hline Not used (500) & 1.00 & & & 1.00 & & \\
\hline Low dose (19) & 0.72 & $0.32-1.62$ & 0.424 & 0.49 & $0.21-1.14$ & 0.096 \\
\hline High dose (16) & 0.41 & $0.13-1.28$ & 0.126 & 0.32 & $0.10-1.00$ & 0.049 \\
\hline \multicolumn{7}{|l|}{ Ping Wei San } \\
\hline Not used (475) & 1.00 & & & 1.00 & & \\
\hline Low dose (41) & 0.83 & $0.47-1.45$ & 0.505 & 0.56 & $0.32-1.01$ & 0.054 \\
\hline High dose (19) & 0.45 & $0.17-1.20$ & 0.110 & 0.31 & $0.11-0.84$ & 0.022 \\
\hline \multicolumn{7}{|l|}{ Zhi Gan Cao Tang } \\
\hline Not used (508) & 1.00 & & & 1.00 & & \\
\hline Low dose (13) & 0.18 & $0.03-1.28$ & 0.086 & 0.19 & $0.03-1.36$ & 0.099 \\
\hline High dose (14) & 0.45 & $0.14-1.40$ & 0.165 & 0.48 & $0.15-1.50$ & 0.204 \\
\hline \multicolumn{7}{|l|}{ Li Zhong Tang } \\
\hline Not used (491) & 1.00 & & & 1.00 & & \\
\hline Low dose (20) & 0.63 & $0.26-1.52$ & 0.302 & 0.47 & $0.19-1.16$ & 0.101 \\
\hline High dose (24) & 0.42 & $0.17-1.03$ & 0.057 & 0.50 & $0.20-1.22$ & 0.130 \\
\hline \multicolumn{7}{|c|}{ Du Huo Ji Sheng Tang } \\
\hline Not used (527) & 1.00 & & & 1.00 & & \\
\hline Low dose (2) & $\mathrm{NC}$ & $\mathrm{NC}$ & $\mathrm{NC}$ & $\mathrm{NC}$ & $\mathrm{NC}$ & $\mathrm{NC}$ \\
\hline High dose (6) & 0.31 & $0.04-2.20$ & 0.240 & 0.47 & $0.07-3.38$ & 0.453 \\
\hline \multicolumn{7}{|l|}{ Qui Pi Tang } \\
\hline Not used (500) & 1.00 & & & 1.00 & & \\
\hline Low dose (17) & 1.21 & $0.54-2.73$ & 0.650 & 1.27 & $0.56-2.87$ & 0.571 \\
\hline High dose (18) & 0.78 & $0.34-1.75$ & 0.544 & 0.26 & $0.11-0.59$ & 0.001 \\
\hline \multicolumn{7}{|c|}{ Huo Xiang Zhen Qi San } \\
\hline Not used (494) & 1.00 & & & 1.00 & & \\
\hline Low dose (22) & 1.31 & $0.67-2.56$ & 0.428 & 1.08 & $0.55-2.14$ & 0.819 \\
\hline High dose (19) & 0.48 & $0.18-1.29$ & 0.145 & 0.66 & $0.24-1.78$ & 0.407 \\
\hline
\end{tabular}

CI: confidence interval; HR: hazards ratio; NC: not calculable.

TABLE 6: Daily dosage and duration of Chinese herbal medicine administration.

\begin{tabular}{lccc}
\hline Formula & Dose & $\begin{array}{c}\text { Daily dosage }^{\mathrm{a}} \\
(\mathrm{g})\end{array}$ & $\begin{array}{c}\text { Duration }^{\mathrm{b}} \\
\text { (days) }\end{array}$ \\
\hline Jia Wei Xiao Yao San & High & 4.59 & 63 \\
Zhi Bai Di Huang Wan & How & 4.09 & 25 \\
Ping Wei San & High & 4.50 & 57 \\
Qui Pi Tang & High & 4.92 & 58 \\
\hline
\end{tabular}

Patients taking dosage above and below the median were classified as

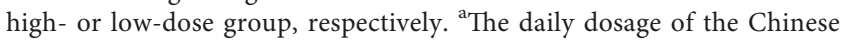
herbal medicine administration was calculated as follows: total dosage/ total days. ${ }^{\mathrm{b}}$ The duration of the Chinese herbal medicine administration required for the high- and low-dose groups was calculated as follows: (median dose/daily dosage of the high-dose group) and (total dosage/ daily dosage of the low-dose group). mellitus were shown to have a significantly shorter overall survival rate [22]. Zhi Bah Di Huang Wan could exhibit a synergistic effect of reducing blood glucose or increasing insulin sensitivity and delaying the insulin resistance of cells $[23,24]$.

Third, patients receiving sphincter-preserving surgery, especially when it is combined with radiotherapy often experience bothersome changes in bowel habits, such as fecal incontinence, frequent bowel movements, urgency, and emptying difficulties, which can lead to a low quality of life $[25,26]$. Pin Wei San could reduce flatulence in the gastrointestinal tract for cancer patients suffering from side effects, such as nausea and vomiting [14].

Fourth, Qui Pi Tang can be used to treat amnesia, fatigue, poor memory or forgetfulness, anorexia, anemia, insomnia, palpitation, and neurosis [27]. It can also treat blood stools, 
subcutaneous purple spots, diet reduction, pain, and severe insomnia [28-30]. Moreover, Qui Pi Tang has been reported to exert antistress effects and beneficial effects on gastrointestinal and immune-mediated diseases [31], which are the common side effects of colorectal cancer after radiotherapy and chemotherapy.

There are a few limitations worth noting in this study. First, the research only chooses to confirm the diagnosis of Western medicine at a single regional hospital, as well as the patients who also receive the CHM treatment in outpatient and consultation. Alternative therapies are common in the United States (45.0-51.8\%) [19, 32] and Norwegian (33.8\%) [33]. There were $27.48 \% \mathrm{CHM}$ users in this study; in contrast, only $12-20 \%$ of colon cancer patients in the NHIRD database receive CHM treatment [34]. However, our research findings are in line with prior studies, showing a higher survival rate and longer survival time for CHM users. Thus, the difference would be reasonably overlooked.

Second, the possibility of selection bias could not entirely be ruled out because $674(55.7 \%)$ out of 1,209 patients identified from the TCR were excluded from the analysis due to missing information on survival status or cancer stage. Nevertheless, comparison of the age and sex distribution between the source population and the study sample did not reveal any significant differences.

Third, CHM use corresponds to TCM syndrome differentiation, yet the treatment by Chinese medicine practitioners is highly individualized. Practically, according to the syndrome types of patients, the prescriptions and the herbal formulae might differ. Syndrome differentiation is diagnosed by Chinese medicine practitioners, lacking a scientific system to provide more delicate cluster analysis. Consequently, it is urgent to apply artificial intelligence to develop automatic TCM syndrome differentiation system [35]. As such, "syndrome type" can be classified by the system, and more accurate CHM use can be obtained for TCM patients. In this case, we could expect to dig deeper into the effectiveness of $\mathrm{CHM}$ use.

Fourth, we focused on colorectal cancer-specific mortality as the outcome of this study. Cause of death due to complications, such as cardiac arrest, at the end-stage of colorectal cancer would not be included in the survival analysis. Nevertheless, differential misclassification, if present, in the cause of death due to complications should not be different between the CHM users and CHM nonusers groups. In addition, since the use of chemotherapy was marginally higher in the CHM users group compared with the CHM nonuser group $(P=0.069)$, it might increase the mortality due to cardiac events in the CHM users group. However, the potential confounding effect of chemotherapy was adjusted in the final multivariate Cox model. Therefore, the use of colorectal cancer-specific mortality should not affect our conclusion.

Despite these limitations, this study has several strengths. The data source of this study was based on a combination of the TCR and hospital clinical data. Therefore, the survival time and the cause of death could be accurately ascertained. In addition, CHM use patients' information, and comorbidities were obtained from the medical record database at a single regional hospital, thereby minimizing coding and misclassification errors.

\section{Conclusions}

This retrospective cohort study showed that integrated CHM and Western medicine was able to significantly improve survival in patients with colorectal cancer. In particular, Jia Wei Xiao Yao San, Zhi Bah Di Huang Wan, Ping Wei San, and Qui Pi Tang were significantly associated with better survival in patients with colorectal cancer. Findings from this study call for additional research on integrating TCM with Western medicine to improve cancer survival and reduce medical expenditures.

\section{Abbreviations \\ AJCC: American Joint Committee on Cancer \\ CHM: $\quad$ Chinese herbal medicine \\ CI: $\quad$ Confidence interval \\ ICD-9- International Classification of Disease, Clinical \\ CM: $\quad$ modification, 9th edition \\ HR: $\quad$ Hazard ratio \\ NHIRD: National Health Insurance Research Database \\ SD: $\quad$ Standard deviation \\ TCM: $\quad$ Traditional Chinese Medicine \\ TCR: Taiwan Cancer Registry.}

\section{Data Availability}

The data used to support the findings of this study are available from the corresponding author upon request.

\section{Conflicts of Interest}

The authors declare that there are no conflicts of interest regarding the publication of this article.

\section{Authors' Contributions}

Ming-Hsien Yeh, Hung-Pin Chiu, Mei-Chun $\mathrm{Wu}$, and Malcolm Koo contributed equally to this work. MHY and TML conceptualized the study. MHY and CCY were involved in the acquisition of data. MHY, HPC, MCW, NWL, and TML interpreted the data. MHY and MK analyzed the data. MHY and TML drafted the manuscript. MK revised the manuscript. All authors have read and approved the final manuscript.

\section{Acknowledgments}

This work was supported by the Tzu Chi Foundation School and Hospital Cooperation Program (no. CHMMP105-11$02)$.

\section{References}

[1] Ministry of Health and Welfare, Cancer Registry Annual Report, Ministry of Health and Welfare, Taipei, Taiwan, 2015. 
[2] H. Brenner and C. Chen, "The colorectal cancer epidemic: challenges and opportunities for primary, secondary and tertiary prevention," British Journal of Cancer, vol. 119, no. 7, pp. 785-792, 2018.

[3] A. Drouillard, A.-M. Bouvier, F. Rollot, J. Faivre, V. Jooste, and C. Lepage, "Conditional net survival: relevant prognostic information for colorectal cancer survivors. A French population-based study," Digestive and Liver Disease, vol. 47, no. 7, pp. 597-601, 2015.

[4] N. P. M. Brouwer, A. C. R. K. Bos, V. E. P. P. Lemmens et al., "An overview of 25 years of incidence, treatment and outcome of colorectal cancer patients," International Journal of Cancer, vol. 143, no. 11, pp. 2758-2766, 2018.

[5] E. J. Kuipers, W. M. Grady, D. Lieberman et al., "Colorectal cancer," Nature Reviews Disease Primers, vol. 1, p. 15065, 2015.

[6] S.-Y. Yin, W.-C. Wei, F.-Y. Jian, and N.-S. Yang, "Therapeutic applications of herbal medicines for cancer patients," Evidence-Based Complementary and Alternative Medicine, vol. 2013, Article ID 302426, 15 pages, 2013.

[7] Z. Wang, F. Qi, Y. Cui et al., "An update on Chinese herbal medicines as adjuvant treatment of anticancer therapeutics," BioScience Trends, vol. 12, no. 3, pp. 220-239, 2018.

[8] P. Zhang, X. Meng, X. Tang, L. Ren, and J. Liang, "The effect of a coix seed oil injection on cancer pain relief," Supportive Care in Cancer, vol. 27, no. 2, pp. 461-465, 2019.

[9] S. Zhang, L. Shi, D. Mao et al., "Use of Jianpi Jiedu herbs in patients with advanced colorectal cancer: a systematic review and meta-analysis," Evidence-Based Complementary and Alternative Medicine, vol. 2018, Article ID 6180810, 13 pages, 2018.

[10] Y.-Y. Lin, I.-Y. Lee, W.-S. Huang et al., "Danshen improves survival of patients with colon cancer and dihydroisotanshinone I inhibit the proliferation of colon cancer cells via apoptosis and skp2 signaling pathway," Journal of Ethnopharmacology, vol. 209, pp. 305-316, 2017.

[11] T.-H. Chao, P.-K. Fu, C.-H. Chang, S.-N. Chang, F. Chiahung Mao, and C.-H. Lin, "Prescription patterns of Chinese herbal products for post-surgery colon cancer patients in Taiwan," Journal of Ethnopharmacology, vol. 155, no. 1, pp. 702-708, 2014.

[12] Y. Han, H. Wang, W. Xu et al., "Chinese herbal medicine as maintenance therapy for improving the quality of life for advanced non-small cell lung cancer patients," Complementary Therapies in Medicine, vol. 24, pp. 81-89, 2016.

[13] H.-R. Yen, W.-Y. Lai, C.-H. Muo, and M.-F. Sun, "Characteristics of traditional Chinese medicine use in pediatric cancer patients: a nationwide, retrospective, taiwanese-registry, population-based study," Integrative Cancer Therapies, vol. 16, no. 2, pp. 147-155, 2017.

[14] Y.-T. Kuo, H.-H. Liao, J.-H. Chiang et al., "Complementary Chinese herbal medicine therapy improves survival of patients with pancreatic cancer in Taiwan: a nationwide population-based cohort study," Integrative Cancer Therapies, vol. 17, no. 2, pp. 411-422, 2018.

[15] A. W. Hsing and J. P. A. Ioannidis, "Nationwide population science: lessons from the Taiwan national health insurance research database," JAMA Internal Medicine, vol. 175, no. 9, pp. 1527-1529, 2015.

[16] C.-J. Chiang, S.-L. You, C.-J. Chen, Y.-W. Yang, W.-C. Lo, and M.-S. Lai, "Quality assessment and improvement of nationwide cancer registration system in Taiwan: a review," Japanese Journal of Clinical Oncology, vol. 45, no. 3, pp. 291-296, 2015.
[17] H.-S. Shen and S.-H. Wen, "Effect of early use of Chinese herbal products on mortality rate in patients with lung cancer," Journal of Ethnopharmacology, vol. 211, pp. 1-8, 2018.

[18] R. Deyo, D. C. Cherkin, and M. A. Ciol, "Adapting a clinical comorbidity index for use with ICD-9-CM administrative databases," Journal of Clinical Epidemiology, vol. 45, no. 6, pp. 613-619, 1992.

[19] M. McCulloch, M. Broffman, M. van der Laan et al., "Colon cancer survival with herbal medicine and vitamins combined with standard therapy in a whole-systems approach," Integrative Cancer Therapies, vol. 10, no. 3, pp. 240-259, 2011.

[20] Y.-Y. Cheng, C.-H. Hsieh, and T.-H. Tsai, "Concurrent administration of anticancer chemotherapy drug and herbal medicine on the perspective of pharmacokinetics," Journal of Food and Drug Analysis, vol. 26, no. 2, pp. S88-S95, 2018.

[21] M.-H. Chiang, L.-W. Chang, J.-W. Wang, L.-C. Lin, and T.-H. Tsai, "Herb-drug pharmacokinetic interaction of a traditional Chinese medicine jia-wei-xiao-yao-san with 5Fluorouracil in the blood and brain of rat using microdialysis," Evidence-Based Complementary and Alternative Medicine, vol. 2015, Article ID 729679, 11 pages, 2015.

[22] K.-H. Chen, Y.-Y. Shao, Z.-Z. Lin et al., "Type 2 diabetes mellitus is associated with increased mortality in Chinese patients receiving curative surgery for colon cancer," The Oncologist, vol. 19, no. 9, pp. 951-958, 2014.

[23] Y. J. Lin, T. J. Ho, Y. C. Yeh et al., "Chinese herbal medicine treatment improves the overall survival rate of individuals with hypertension among type 2 diabetes patients and modulates in vitro smooth muscle cell contractility," PLoS One, vol. 10, no. 12, Article ID e0145109, 2015.

[24] C. T. Wu, Y. T. Tsai, J. G. Lin, S. L. Fu, and J. N. Lai, "Chinese herbal products and the reduction of risk of breast cancer among females with type 2 diabetes in Taiwan: a case-control study," Medicine (Baltimore), vol. 97, no. 31, Article ID e11600, 2018.

[25] T. Y. Chen, K. J. Emmertsen, and S. Laurberg, "Bowel dysfunction after rectal cancer treatment: a study comparing the specialist's versus patient's perspective," BMJ Open, vol. 4, no. 1, Article ID e003374, 2014.

[26] T. Juul, M. Ahlberg, S. Biondo et al., "Low anterior resection syndrome and quality of life," Diseases of the Colon \& Rectum, vol. 57, no. 5, pp. 585-591, 2014.

[27] Y. Lee, Y.-R. Lee, C.-S. Kim et al., "Effect of Guibi-tang, a traditional herbal formula, on retinal neovascularization in a mouse model of proliferative retinopathy," International Journal of Molecular Sciences, vol. 16, no. 12, pp. 29900-29910, 2015.

[28] L. Liu, C. Liu, Y. Wang, P. Wang, Y. Li, and B. Li, "Herbal medicine for anxiety, depression and insomnia," Current Neuropharmacology, vol. 13, no. 4, pp. 481-493, 2015.

[29] W.-F. Yeung, K.-F. Chung, K.-Y. Ng et al., "Prescription of Chinese herbal medicine in pattern-based traditional Chinese medicine treatment for depression: a systematic review," Evidence-Based Complementary and Alternative Medicine, vol. 2015, Article ID 160189, 12 pages, 2015.

[30] T. Fleischer, T.-T. Chang, J.-H. Chiang, M.-F. Sun, and H.-R. Yen, "Improved survival with integration of Chinese herbal medicine therapy in patients with acute myeloid leukemia: a nationwide population-based cohort study," Integrative Cancer Therapies, vol. 16, no. 2, pp. 156-164, 2017.

[31] H. Park, Y.-H. Hwang, H. J. Yang, H.-K. Kim, K. S. Song, and J. Y. Ma, "Acute toxicity and genotoxicity study of fermented traditional herb formula Guibi-tang," Journal of Ethnopharmacology, vol. 156, pp. 182-189, 2014. 
[32] C. A. Buckner, R. M. Lafrenie, J. A. Dénommée, J. M. Caswell, and D. A. Want, "Complementary and alternative medicine use in patients before and after a cancer diagnosis," Current Oncology, vol. 25, no. 4, pp. e275-281, 2018.

[33] A. E. Kristoffersen, A. J. Norheim, and V. M. Fønnebø, "Complementary and alternative medicine use among Norwegian cancer survivors: gender-specific prevalence and associations for use," Evidence-Based Complementary and Alternative Medicine, vol. 2013, pp. 1-10, 2013.

[34] Y.-H. Liao, C.-I. Li, C.-C. Lin, J.-G. Lin, J.-H. Chiang, and T.-C. Li, "Traditional Chinese medicine as adjunctive therapy improves the long-term survival of lung cancer patients," Journal of Cancer Research and Clinical Oncology, vol. 143, no. 12, pp. 2425-2435, 2017.

[35] P. Gu, H. Chen, and T. Yu, "Ontology-oriented diagnostic system for traditional Chinese medicine based on relation refinement," Computational and Mathematical Methods in Medicine, vol. 2013, Article ID 317803, 11 pages, 2013. 


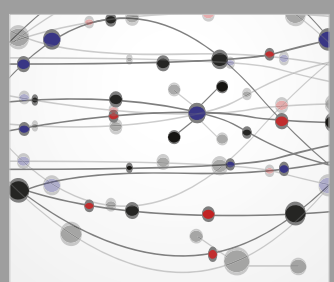

The Scientific World Journal
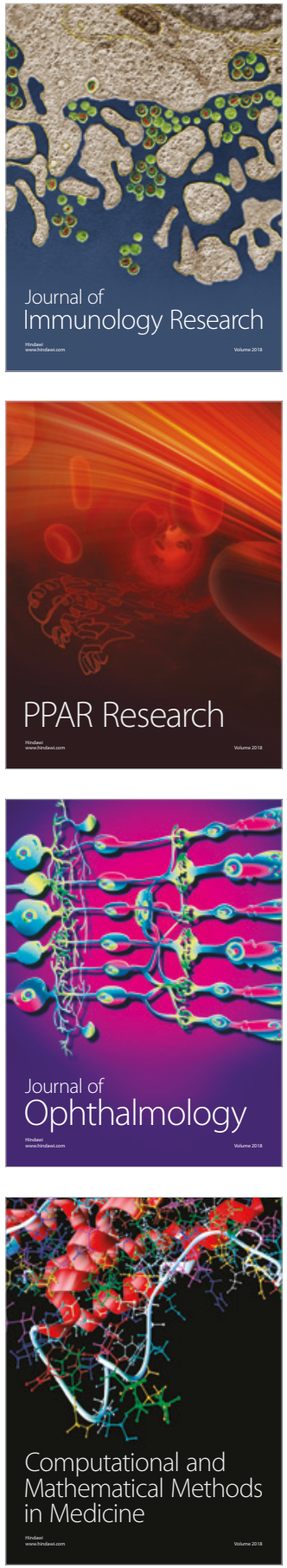

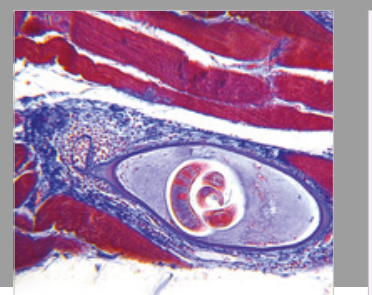

Gastroenterology Research and Practice

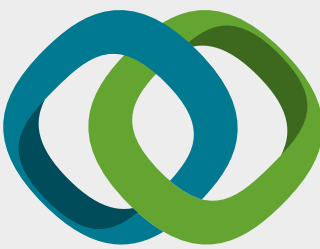

\section{Hindawi}

Submit your manuscripts at

www.hindawi.com
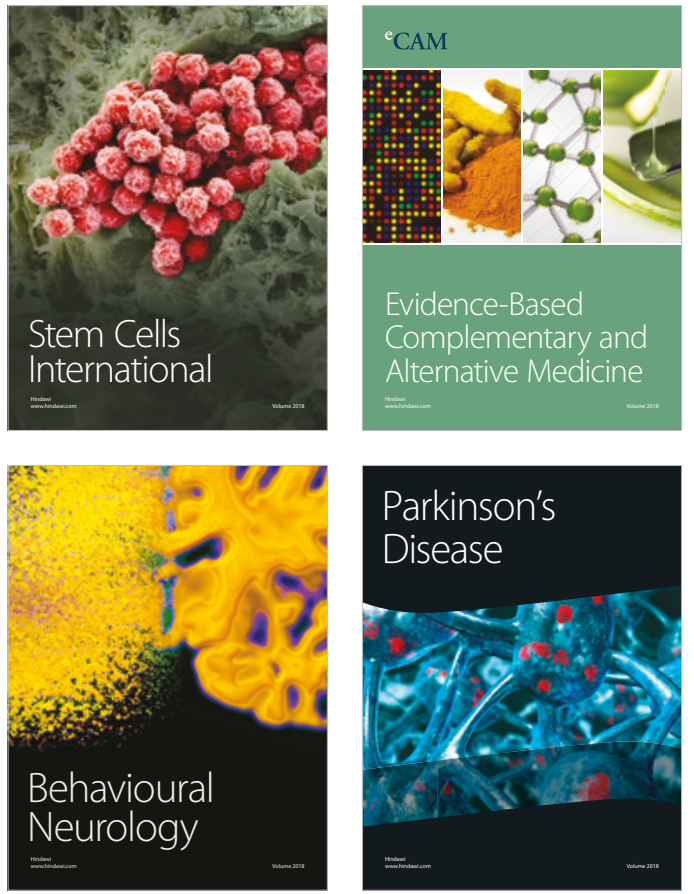

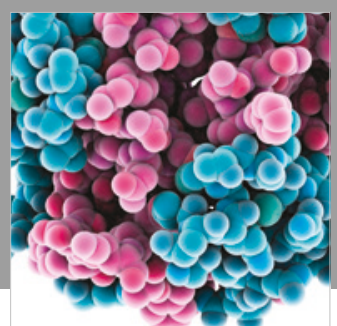

ournal of

Diabetes Research

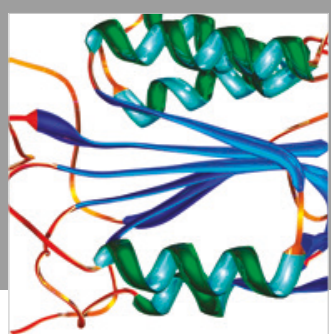

Disease Markers
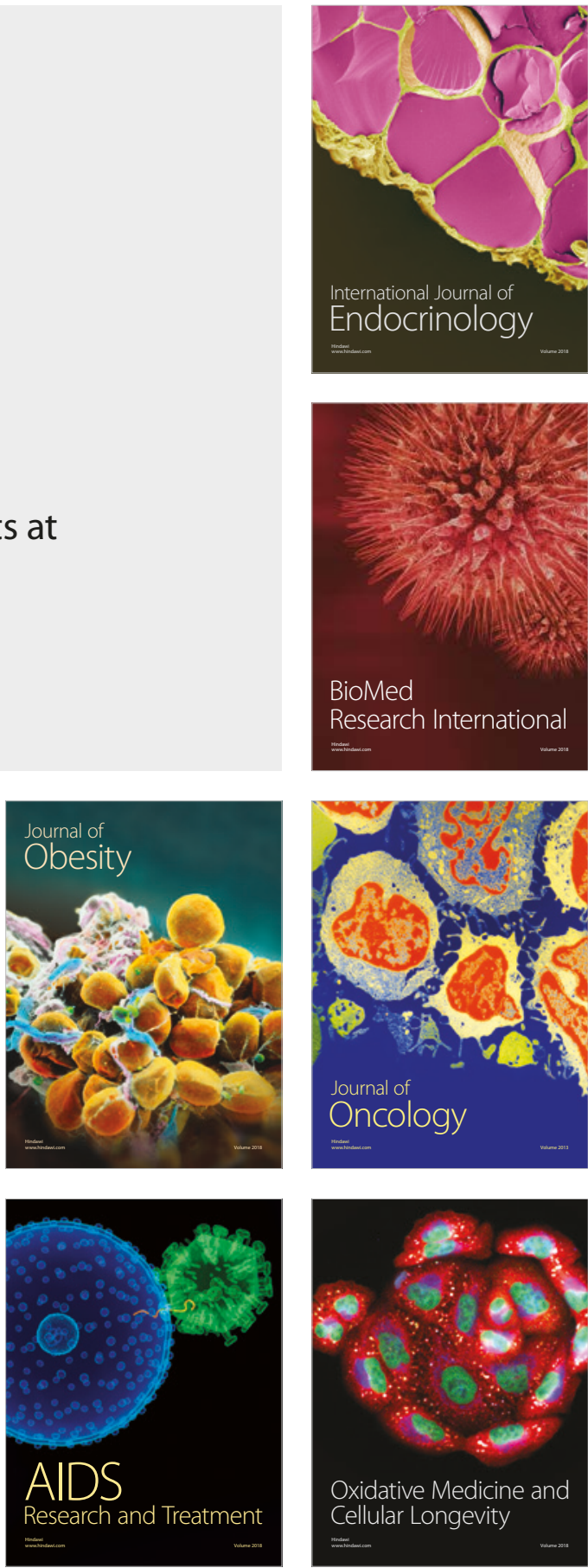